\title{
ARTALIVE: AN ANDROID APPLICATION FOR AUGMENTED REALITY WITHOUT MARKERS, BASED ON ANAMORPHIC IMAGES
}

\author{
A. Alvaro-Tordesillas ${ }^{1 *}$, S. Crespo-Aller ${ }^{1}$, S. Barba ${ }^{2}$ \\ ${ }^{1}$ ETS Architecture of Valladolid, University of Valladolid, Spain - tordesillas@ arq.uva.es \\ ${ }^{2}$ Dep. of Civil Engineering, Faculty of Engineering, University of Salerno, Italy - sbarba@ unisa.it
}

Commission II, WG II/8

KEY WORDS: Augmented Reality, Anamorphosis, Projective Geometry, Mobile Application, Unity, Vuforia, Museum experience

\begin{abstract}
:
The project that we present develops a mobile application (ArtAlive $\left.{ }^{\circledR}\right)$ for the generation of augmented reality experiences on museum sculptural objects. The novelty of our application is that we eliminate the markers that other applications need to run. We do this turning the sculptures into their own markers, based on the recognition of anamorphic images of these sculptures: twodimensional images of three-dimensional objects. So we eliminate the intermediate elements of the process making the experience easier. The aim is to transform the experience of visiting a museum into a more interactive and intuitive experience. The possibility of accessing the information contained in the augmented reality experiences linked specifically to each work, from the comfort of the smartphone, gives the process greater accessibility and flexibility.

The recognition of the objects is developed with Vuforia, an augmented reality software development kit (SDK) for mobile devices. ArtAlive ${ }^{\circledR}$ has been registered in the Spanish Intellectual Property Registry, and a possible business model has been defined in a very basic way.
\end{abstract}

\section{INTRODUCTION}

\subsection{Augmented Reality}

Augmented Reality (AR) is the set of technologies that allow a user to jointly visualize part of the real world and added graphic information through a technological device. This device (smartphones, tablets, etc.) adds virtual information to the existing physical information, in such a way that tangible physical elements are combined with virtual elements thus creating an augmented reality in real time. The most attractive part of this concept of augmented reality is, precisely, the inclusion of the real world as an environment, without the need to abstract to an unreal world, making ourselves more involved in the experience of that reality.

\subsection{The project and its scientific interest}

The AR aims to become a common reality, seeking to make room in large areas of daily life, such as education, leisure and work, in countless labour camps, as well as in almost any branch of scientific research. However, we believe that this will happen when the process is simpler. To date, all AR experience requires the intermediation of some 'markers' between the user and the object to be visualized. In an attempt to simplify this process, our proposal focuses on eliminating these intermediate markers; and we do it by converting the objects themselves into markers. With this, the mere visualization of an object triggers the AR experience of that object.
The project also presents an application to install on users' devices, which allows the recognition of objects and the AR experience of each of them.

\subsection{Previous works}

Prior to the research, an analysis of the existing technologies with similar objectives has been carried out, up to now. Thus, in the current scenario, it is possible to find numerous projects of this nature, including the recognition of images by computer (Redmon, 2017), as augmented reality and virtual reality are two fields belonging to the next technological revolution.

Among all the existing research, it is worth highlighting some developments, such as the HideOut project (Willis, Shiratori and Mahler, 2013). This research project, developed by a compendium of companies including Disney $\odot$, Microsoft $\odot$ and the Computational Design Lab, at Carnegie Mellon University, also involves the elimination of intermediate markers using invisible ink to the human eye, detected through specific readers.

On the other hand, Google $\odot$ is one of the strongest market bets in the research and development of this technology. It has numerous projects, among which include the following:

Google Lens, an application that allows the recognition of type-objects (such as commercial items) and their association with similar objects, information about them, other similar products, etc.

\footnotetext{
* Corresponding author
} 
- $\quad$ ARCore is a platform for building augmented reality experiences (Google, 2019). Using different Android and iOS's APIs, ARCore enables digital dispositive to sense its environment, understand the world and interact with information. ARCore is based on the previous Project Tango, that used computer vision to enable mobile devices to detect their position relative to the world around them without using GPS or other external signals (Google and ATAP, 2017). Project Tango was abandoned at the end of 2017.

With ARCore, Google $\odot$ is positioned in direct competition with another similar project launched by Apple $($, Arkit, for iOS mobile devices. ARKit combines device motion tracking, camera scene capture, advanced scene processing, and display conveniences to simplify the task of building an AR experience (Apple, 2019). Environments captured through the device can have animated $3 \mathrm{D}$ virtual text, objects and characters added to them.

\section{THE PROJECT}

The project that we present in this Congress develops a mobile application for smart devices whose objective is the generation of augmented reality experiences on sculptural museum objects. It seems obvious the interest of the project to introduce augmented reality, by the hand of our application, in museum exhibitions of sculptural works of art. In fact, after the research and development of the application, a test was made with some pieces of the permanent exhibition of the Jorge Oteiza MuseumFoundation, in Alzuza (Pamplona), in Spain.

\subsection{Objective}

The novelty and particularity of our application is that, although augmented reality currently needs markers to work with the mobile devices that make it possible, we propose a method that eliminates them, transforming the own sculpture into the marker. To do this, we base the recognition of these mobile devices on anamorphic silhouettes of the objects of physical reality. That is, we eliminate the intermediate elements of the process.

The technology we develop consists in focusing with a smartphone towards a sculptural work of art, obtaining on the screen the direct vision of this sculpture, plus personalized virtual information linked to that work. This information will be completely updateable.

It seeks to transform the experience of visits to a museum, or other exhibition surfaces, into a more interactive and intuitive experience. The possibility of accessing the information contained in the augmented reality experiences linked specifically to each work, from the comfort of the smartphone, gives the process greater accessibility and flexibility, compared to the need to use auxiliary elements such as posters, audio guides, QR codes, etc. that, in fact, only they represent a break in the understanding of a piece of sculpture.

\subsection{Methodology}

The main hypothesis of this project is that we can convert any three-dimensional object into a two-dimensional image of itself. This hypothesis becomes the objective of the project by allowing us to create augmented reality experiences from real objects, eliminating any intermediate element of the process; so converting the object itself into a marker (fig. 1). It is about creating an augmented reality experience whose operation is based on the optical recognition of images or codes but does not require a code as such, rather this is the element itself the generator of augmented reality experience. This hypothesis is based, and is possible, thanks to the current degree of development of augmented reality technology.
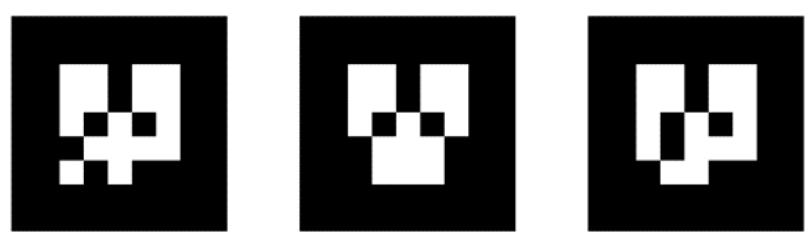

Figure 1. Classical markers for AR

Theoretically, the project is based on the possibility of deceiving both the human eye and a camera of any electronic device, making them believe that they see a two-dimensional object when in reality they look at a three-dimensional one. This is described and belongs to the field of knowledge of Descriptive Geometry, specifically Projective Geometry, and is known as Anamorphosis (fig. 2 and 3) (Brannan, Esplen and Gray, 1999; Cabezos Bernal, Cisneros Vivó and Soler Sanz, 2014).

The Anamorphosis is a distorted projection or perspective requiring the viewer to occupy a specific vantage point, use special devices or both to view a recognizable image.

This does not mean that has been developed a parallel geometry to deceive the device, but that the same has been used, modifying it for a determined point of view, which allows us to establish this relationship between the device and the object from an appropriate position for both user and the object itself.

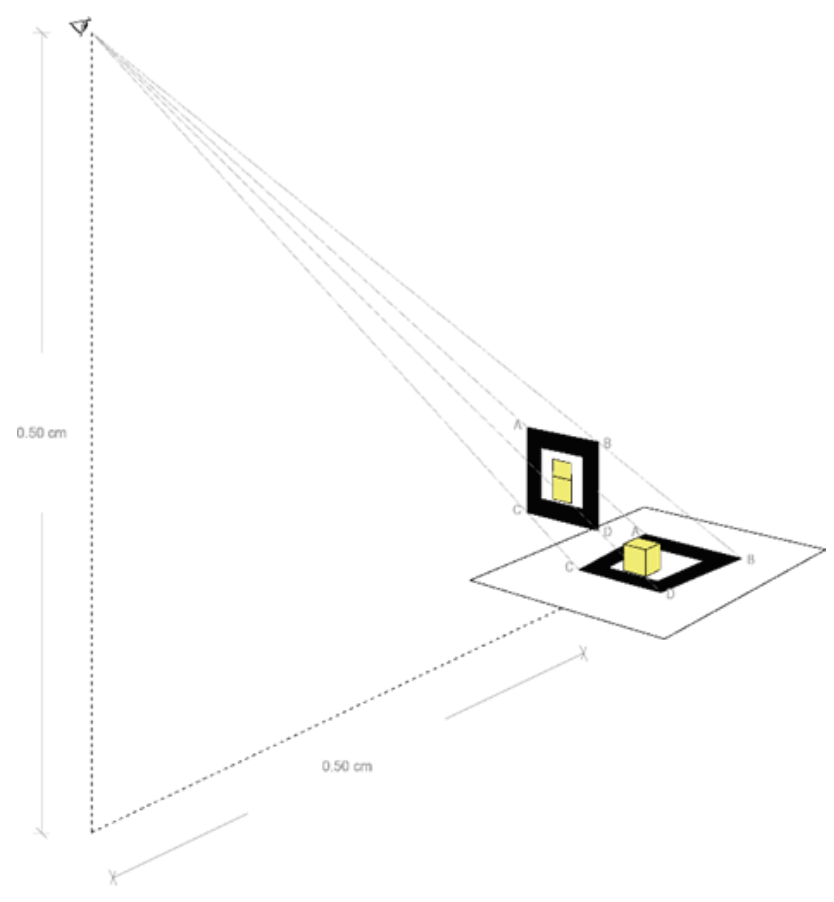

Figure 2. Geometry of anamorphosis 

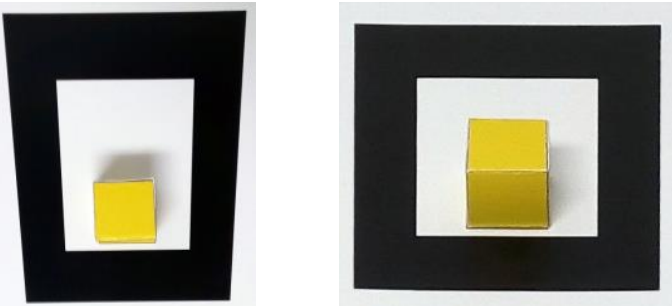

Figure 3. Plan view of the real object (left) versus image used as a marker (right)

Methodologically the project is developed in three ways:

a. To find the best variables to produce a simple anamorphosis: distance to the object, height of the camera, lighting conditions, focus, etc.

b. An analysis of the recognition of images with Vuforia, an augmented reality software development kit (SDK) for mobile devices. It uses computer vision technology to recognize and track planar images (image markers) and simple 3D objects, such as boxes, in real time. This process is also called Image Tracking, a process by which the device camera detects a predetermined image or object and knows what to do with it, such as rendering some content on top of it.

c. Development of an own application for the recognition of objects and launching of the $\mathrm{AR}$ in the device.

In all of them, we have experimented with different objects, based on four characteristics: colour, size, geometric regularity and additional objects.

\subsection{Results}

The results obtained are clearly positive. We are going to analyze them according to the three paths followed in the methodology.

\subsubsection{Conditions for a simple anamorphosis.}

The variables that determine a simple anamorphic image have been tested in conditions similar to those we might find in a museum room. Thus, the distance to the object is determined by the base on which the sculpture is located, having a range of distances between 50 and $100 \mathrm{~cm}$; and the height depends on the person who uses the application, taking into account that it is not the eye, but the camera held by both hands at $120 \mathrm{~cm}$ from the ground, on average.

The conditions of light do not determine the anamorphic image of the object, as long as it is white; although it has been noted how spotlights generate very aggressive shadows, which can confuse the image of the object with the background on which it is placed.

The focal length of camera lenses of mobile devices generally moves in the range of $3.43 / 5.14 \mathrm{~mm}$; which for sensors of an average size of $1 / 2.3 "$ and a crop factor of 5.83, suppose distances of $20 / 30 \mathrm{~mm}$; that is, great angular vision.
2.3.2 Results of the Image Tracking. To check the results of the developed Image Tracking, we work with four types of objects of different characteristics and situations. Table 1 presents a summary of the results obtained for each group of objects:

\begin{tabular}{|c|c|}
\hline Tests & Results \\
\hline Chromatic differences & + \\
\hline Irregular geometries & + \\
\hline Additional objects & - \\
\hline Real sculptural pieces & + \\
\hline
\end{tabular}

Table 1. Positive (+) or negative (-) results of Image Tracking developed with Vuforia SDK, for the four groups of proposed objects

In the first group we used a variety of objects of identical geometry but with different colours, playing with the three variables of colour: hue, saturation and brightness. The results were positive, showing recognition only in the case of colour matching. This means that the recognition not only looked at geometry as such, but also at its colours. This result supposes a slight conditioner in the importance of the illumination used in the processes of recognition and transformation of the object in its marker.

In the second group, we used a selection of objects with different geometries, with different degrees of irregularity. The results are positive in the sense that the recognition occurs correctly, rejecting the recognition when the geometry does not adapt completely to the determined marker. This implies that it does not matter how complicated the geometry of the object is.

In this process of testing the possibilities of anamorphosis with respect to the recognition of images, the question arose as to what would happen if objects were introduced next to or superimposed on the object to be recognized. In both cases, the result is negative, because there is an interruption of the recognition given that the vision of the device on the target is modified radically. It seems that this may be a point to develop over time, trying to make the recognition stable even if it is interrupted, as we have seen.

Finally, the fourth group includes some real pieces from the Museum-Fundación Jorge Oteiza collection, although they were not included in the initial methodology; that show the possibilities of this project (fig. 4).

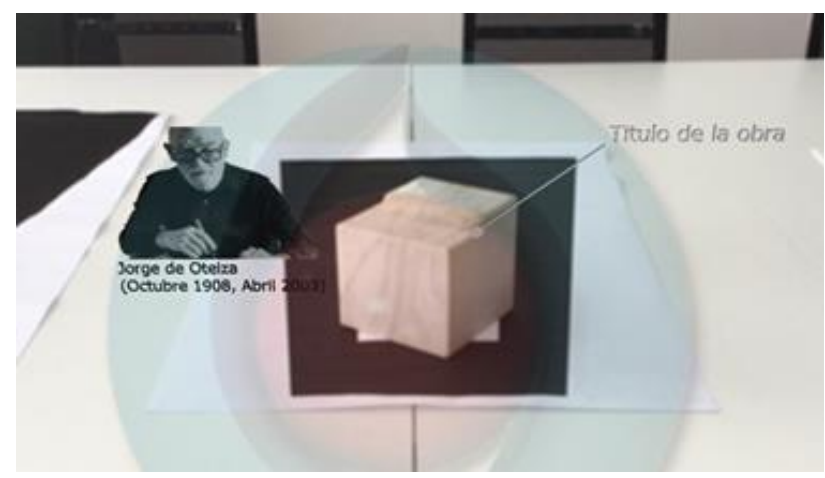

Figure 4. Experience of Augmented Reality on a sculpture by Jorge de Oteiza (Museo Jorge Oteiza, Navarra, Spain) 
The inclusion of these objects is given with the intention of studying the behaviour of this technique with real objects, outside of a clean laboratory environment. Although, this does not mean that these results can be extrapolated to any environment.

\subsubsection{A prototype of own mobile application: ArtAlive ${ }^{\circledR}$.}

The need to use different external software to carry out the whole process takes us to the next step, which also, due to the special characteristics of our project, is also the next logical step in this investigation. This consists in the development of a mobile application prototype that allows the generation of augmented reality experiences on some sculptural works exhibited in the Jorge Oteiza Museum-Foundation; being themselves the ones that, from determined points of view, launch the augmented reality on the mobile devices arranged.

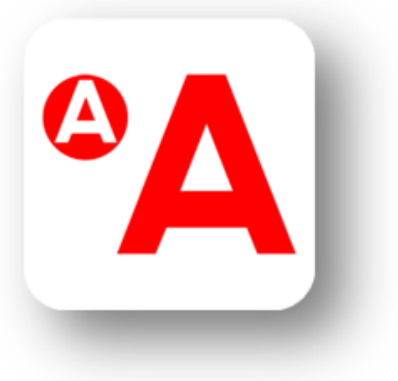

Figure 5. Application logo 'ArtAlive ${ }^{\circledR}$ '

This application, with the name of ArtAlive ${ }^{\circledR}$ (fig. 5), has been developed in programming language $\# \mathrm{C}$ for the source code of the multiplatform application in the Unity Environment 2017.3.0f3 with the free Vuforia v.7 plugin (Unity Technologies, 2017). It is compatible with devices which support Android 2.3.3 or higher.

This application, which is intended to offer a service to different museums as a support element for visits, will logically allow the personalization of the content in the near future, thus adapting to each museum entity; with different possibilities within it: different catalogues, updates of these, etc.

Figure 6 shows the deployment diagram of the application developed. 'UserDevice' represents a device in which the 'ArtAlive ${ }^{\circledR}$ ' application is installed. It must be pointed out that the application uses a camera for its functionality, so the application depends on it for its use, that is to say at least the user device has to have it. To obtain the results, we compare the VuforiaModel of the marker figures of the application (the museum sculpture) with the real object, 'FigureObject' of the second box of figure 6 , which is recognized by the camera.

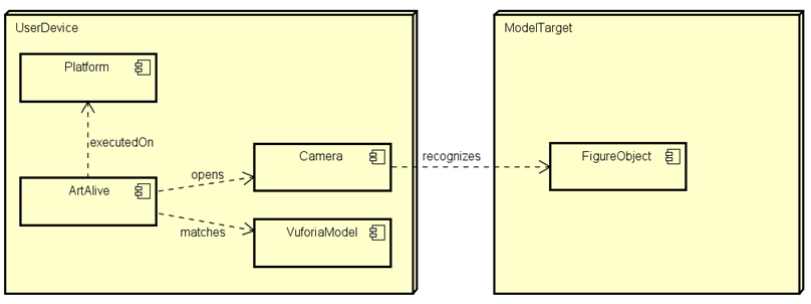

Figure 6. Deployment diagram multiplatform application
Although it is a basic deployment diagram, it clearly establishes the process by which the app is governed. The comparison of the object perceived by the camera with the stored object, produced from its study with the Vuforia technology, is what allows the operation of the app.

The process of obtaining the "Model Marker" of Vuforia is possible through the tools that the technology itself facilitates. Thus, it has a tool that is free access, called "Vuforia Model Marker Generator", which allows the conversion of any 3D object into an object recognizable by the application, "Vuforia Engine Dataset". These recognizable objects are exported in any of the following formats:

$$
\begin{aligned}
& \text { - Creo View (.pvz) } \\
& \text { - Collada (.dae) } \\
& \text { - FBX (.fbx) } \\
& \text { - IGES (.igs, .iges) } \\
& \text { - Wavefront (.obj) } \\
& \text { - STEP (.stp, .step) } \\
& \text { - STL (.stl, .sla) } \\
& \text { - VRML (.wrl, .vrml) }
\end{aligned}
$$

The creation of these models is done by taking convergent digital photographs (fig. 7), according to the principles of digital photogrammetry and described in any manual (Waldhäusl and Ogleby, 1994). And its subsequent processing by any specific software (fig. 8), such as Photoscan or Metashape, by Agisoft SLL, or Photomodeler, by Eos Systems Inc.

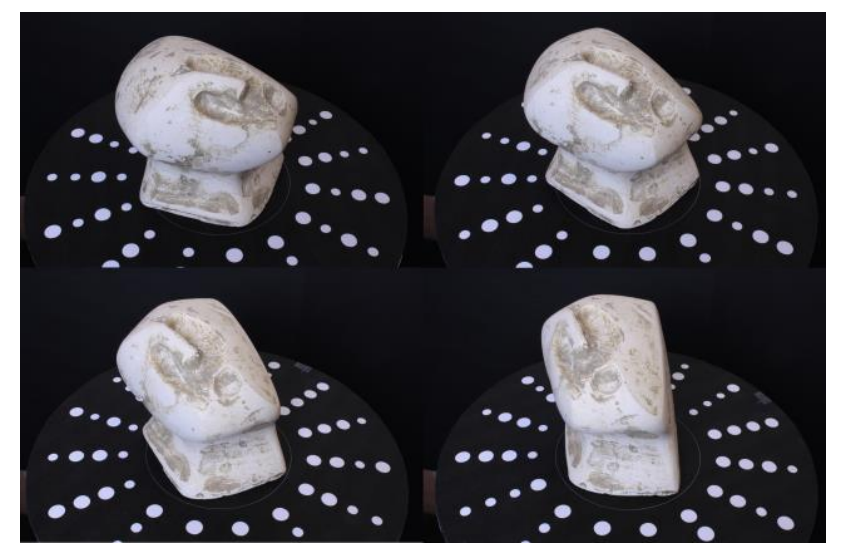

Figure 7. Recreation conditions and scanner.

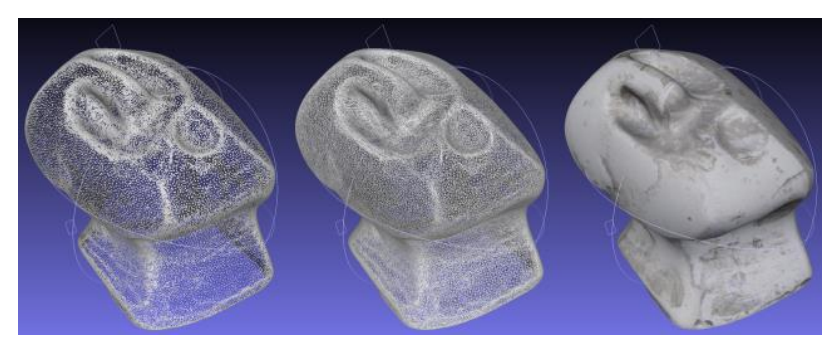

Figure 8. Result of the scanner with obtaining the point cloud and the mesh of the object. 
Finally, the general flow diagram of the application itself is presented (fig. 9):

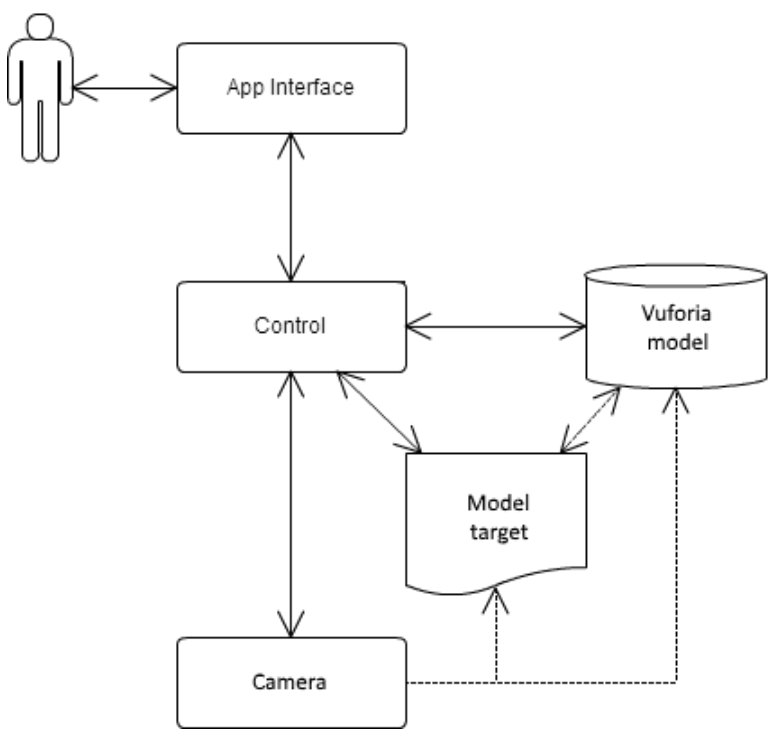

Figure 9: Conceptual flow diagram of the system

The "Control" module contains all the operating logic of the application, in addition to being responsible for continuously updating the interface with the relevant information.

The "Vuforia model" module is the 3D modelling of the real object. This modelling must be very precise since the "Model Target" will be compared with the real object, using the device camera.

The modus operandi of Vuforia "Target Model" allows the application ArtAlive ${ }^{\circledR}$ to do something more than initially raised in this research:

a. The recognition of a stored $2 \mathrm{D}$ image of an object, to trigger the AR experience, based on the anamorphosis principle described.

b. That the physical objects are recognized and tracked using a digital 3D model of the object because they admit the recognition and tracking of the object by its shape. The objects are recognized by the Vuforia SDK using a specially prepared database, which is generated by processing a digital 3D representation of the object using the "Model Marker Generator" application, as we have seen. It is very important to know the position of the figure in the scanned 3D model; it must also be as isolated as possible from other objects around it, as well as having a background colour different from that of the figure.

Figure 10 shows the application in use. Left, it shows the camera of the device waiting to recognize some object; centre, after focusing the object, the information obtained from comparing a Vuforia object with the real image appears; right, the information is maintained if we move the camera to another angle, without focusing on the objective.
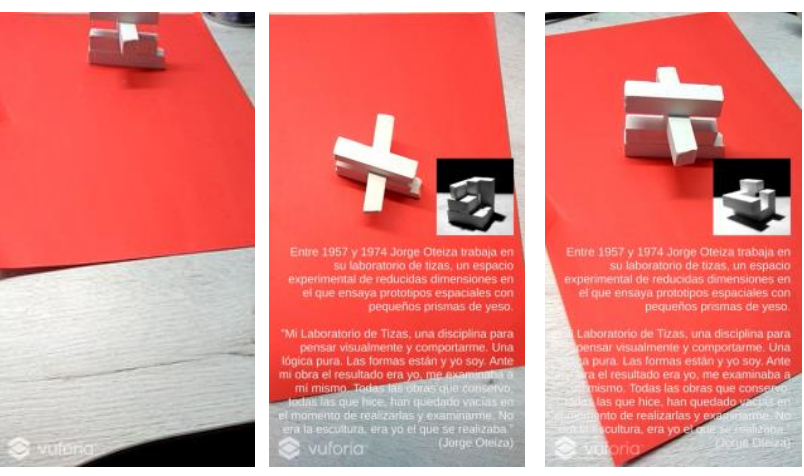

Figure 10. Images of the application working

The project (and the application) has been registered in the Spanish Intellectual Property Registry, and a possible business model has been defined in a very basic way (fig. 11) that establishes the most important parameters for commercial development of the project, as indicated in point 4 .

\section{CONCLUSIONS}

The data obtained indicate that it is possible to carry out an AR experience without the need for intermediate markers.

This project proves how anamorphosis is a valid and efficient technique to simulate 2D images, equivalent to markers for $\mathrm{AR}$, from 3D scenarios. Thanks to this, we developed an Android application that recognizes these simulated two-dimensional images, through the camera of our digital devices (smartphones, tablets) and shoots on its screens the added information that turns the experience into augmented reality. This novelty allows making disappear the typical markers of recognition of the AR. But also, we have seen how the Vuforia development software also allows us, once established the connection between the saved 2D image and the one captured by the camera, to continue with the recognition of the object even moving the camera, thanks to the $3 \mathrm{D}$ recognition of the object itself.

The tests carried out lead to the certainty of its application in museums or exhibitions, allowing easily the deployment of augmented reality experiences whose level of involvement with the work exceeds the typical audio-guide or printed brochure. The possibility of converting the works of art into their own markers, converts the object-information association into the basis of the interaction between the object and the user, facilitating a very intuitive experience.

\section{FUTURE WORKS}

It is clear that the application requires improvement implementations, specifically in the recognition of additional objects, as we saw in point 2.3.2.

In another area, we are beginning to develop a business model that aims to lay the foundations of exploitation of this project, providing different ideas and strategies, and the main lines on which this business should develop. In this plan, important terms are established for such development, such as the value proposals, the possible partners, the clear determination of who the client is and who is the user, something important in this type of project, etc. (fig. 11). 


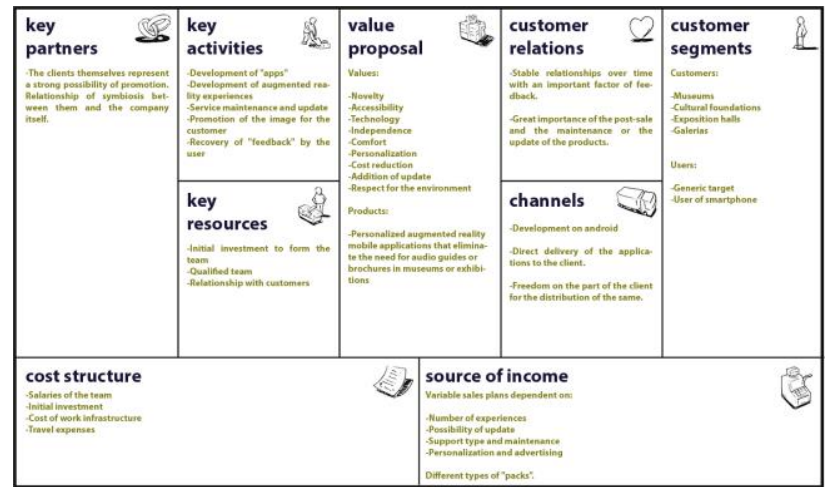

Figure 11. Canvas business model.

\section{ACKNOWLEDGEMENTS}

We gratefully acknowledge support from the Museo-Fundación Jorge Oteiza in Pamplona (Spain). And the generosity and experience in programming Unity of Cristian Tejedor-García, of the School of Computer Engineering, of the University of Valladolid.

\section{REFERENCES}

Apple, 2019. ARKit, Apple Developer Documentation. Available at: https://developer.apple.com/documentation/arkit (Accessed: 25 June 2019).

Brannan, D. A., Esplen, M. F., Gray, J., 1999. Geometry. Cambridge, Cambridge University Press.

Cabezos-Bernal, P., Cisneros-Vivó, J., Soler-Sanz, F., 2014. Anamorfosis, su historia y evolución, EGA Revista de Expresión Gráfica Arquitectónica, 19(23), 148-161. doi: 10.4995/ega.2014. 2184.

Google, 2019. ARCore. Available at: https://developers.google.com/ar/ (Accessed: 17 June 2019).

Google and ATAP, 2017. Tango (platform), Wikipedia. Available at: https://en.wikipedia.org/wiki/Tango_(platform) (Accessed: 17 October 2018).

Redmon, J., 2017. How computers learn to recognize objects instantly | Joseph Redmon - YouTube, TED. Available at: https://www.youtube.com/watch? $=$ Cgxsv1riJhI\&t= (Accessed: 25 June 2019).

Unity Technologies, 2017. Vuforia v.7 plugin. Available at: https://developer.vuforia.com/downloads/sdk (Accessed: 17 June 2019).

Waldhäusl, P., Ogleby, C., 1994. 3-by-3- Rules for Simple Photogrammetric Documentation of Architecture. International Archives of Photogrammetry and Remote Sensing, Volume XXX, Part 5, Melbourne, 1994, 426-429.

Willis, K. D. D., Shiratori, T., Mahler, M., 2013. 'HideOut: Mobile Projector Interaction with Tangible Objects and Surfaces'. International Conference on Tangible, Embedded and Embodied Interaction. Barcelona, 331-338. 